\title{
A la mesa con Magallanes, Elcano y Pigafetta
}

\author{
To the table with Magallanes, Elcano and Pigafetta
}

\author{
María Soledad Aguilar Domingo \\ maria.aguilardomingo@unibo.it \\ Università di Bologna- Forlì \\ María Enriqueta Pérez Vázquez \\ maria.perez@unipi.it \\ Università di Pisa
}

\begin{abstract}
Resumen: Este artículo está dedicado al análisis de las dificultades de interpretación, y por consiguiente de traducción, que representan los términos relacionados con la comida en la Relazione del primo viaggio attorno al mondo, obra en la que Antonio Pigafetta narra la primera circunnavegación del globo terráqueo. En la primera parte del estudio se describe el manuscrito que narra la primera circumnavegación del globo terráqueo y los objetivos de esta. En la segunda parte se examinan los términos relacionados con la comida en la Relación y en la tercera se analizan las dificultades y los problemas de traducción que han ocasionado algunos términos relacionados tanto con los alimentos embarcados en el avituallamiento inicial de las naves como con las comidas exóticas de los lugares en los que van recalando las naves a lo largo de los casi tres años que duró la expedición. Nuestra traducción (en prensa) y el estudio traductológico que aquí presentamos se basa en la edición comentada que realizó Andrea Canova en 1999. Los números que preceden cada uno de los párrafos también pertenecen a la edición de dicho texto.
\end{abstract}

Palabras clave: Pigafetta, traducción, comida, terminología, léxico gastronómico.

Abstract: This article is dedicated to the analysis of the difficulties of interpretation, and therefore of translation, represented by the terms related to food in the Relazione del primo viaggio attorno al mondo, a work in which Antonio Pigafetta narrates the first circumnavigation of the globe. The first part of the study describes the manuscript that narrates the first circumnavigation of the globe and its objectives. The second part examines the terms, locutions, etc. of the food in the Relacion, and the third part analyses the difficulties and translation problems caused by some of the terms related both to the food supplied in the initial provisioning of the ships and to the exotic foods of the places where the ships made landfall during the almost three years that the expedition lasted. Our translation (in press) and the translatological study presented here is based on the annotated edition produced by Andrea Canova in 1999. The numbers preceding each paragraph also belong to the edition of that text.

Keywords: Pigafetta, translation, food, terminology, gastronomic lexicon. 
$\mathrm{M}^{\mathrm{a}}$ Soledad Aguilar Domingo \& $\mathrm{M}^{\mathrm{a}}$ Enriqueta Pérez Vázquez. A la mesa con Magallanes, Elcano

y Pigafetta

\section{Introducción}

\subsection{El viaje narrado}

Desde septiembre de 2019 hasta septiembre de 2022 están teniendo lugar las celebraciones para conmemorar el $\mathrm{V}$ centenario de la primera circunnavegación del mundo que llevaron a cabo Fernando de Magallanes y Juan Sebastián Elcano.

La expedición, que partió de Sevilla el 20 de septiembre de 1519 con cinco naves y 238 hombres, tras tres años de navegación, regresó a Sanlúcar de Barrameda reducida a una sola nave y 18 hombres agotados, famélicos, enfermos y desnutridos. Habían culminado la mayor proeza náutica de la historia de la humanidad al circunnavegar el globo navegando siempre hacia occidente. Recorrieron más de 14.000 leguas arribando a América y costeándola encontraron el estrecho que más tarde se llamaría de Magallanes; atravesaron el Océano Pacífico perdiendo parte de la tripulación a causa del hambre y las enfermedades. Navegaron por varios archipiélagos como las islas Marianas y las Filipinas alcanzando, al fin, su objetivo, las Islas de las Especias, las Molucas, en la actual Indonesia.

La expedición se organizó no solo con afanes económicos, lo cual implicaba la búsqueda de las apreciadas especias como el clavo, sino que también suponía todo un entramado de afanes culturales, religiosos y políticos, que no se cuentan en la obra, donde leemos, más que nada, las aventuras y desaventuras, el hambre y la lucha por una supervivencia basada, durante muchos meses, solo gracias al arroz hervido con agua de mar.

\subsection{El manuscrito}

Son numerosos los avatares que sufrieron los tripulantes de la expedición, pero también son muchas las vicisitudes que ha atravesado la Relación de Pigafetta hasta nuestros días. La historia la conocemos gracias al manuscrito Relazione del primo viaggio attorno al mondo que escribió, con la ayuda de sus apuntes, el vicentino Antonio Pigafetta al regresar a Italia, porque el Diario que fue escribiendo día a día, durante los casi tres años que duró la expedición, cuenta él mismo en esta obra, se lo regaló a Carlos $\mathrm{V}$, cuando llegó a España después de su aventura y que a día de hoy sigue extraviado. Después, una vez ya en Italia, Pigafetta trató de publicar el libro en el que narra la circunnavegación, aunque sin mucho éxito como indica Canova (Aguilar; Canova; Pérez; Valladares, en prensa), pero a partir de ese texto italiano se hicieron dos traducciones al francés diferentes. ${ }^{1}$

En 1797 Carlo Amoretti descubrió en la Biblioteca Ambrosiana de Milán la copia del texto original, jamás publicado por Pigafetta, conocido como Codice Ambrosiano y él mismo realiza una refundición de la obra que no siempre es fiel al original. Hasta aquel momento, la Relación que circulaba en italiano era una retrotraducción del francés.

El texto cuenta con cuatro vocabularios exóticos incluidos por Pigafetta en el relato, que

1 Una editada por Simon de Colines y otra que nos ha llegado en tres manuscritos del siglo XVI, dos de los cuales se encuentran en la Biblioteca Nacional de Francia y el tercero en New Haven en la Beinecke Library de la Universidad de Yale.

SCRIPTA, Revista internacional de literatura i cultura medieval i moderna, núm. 18 / desembre 2021 / pp. 182-194 ISSN: 2340-4841 · doi:10.7203/SCRIPTA.18.22773 
constituyen una de las primeras listas europeas de este tipo: un elenco de palabras y expresiones del «brasileño», del «patagónico», del «filipino»y del «indonesio». El método empleado por Pigafetta para la confección de estos vocabularios parece que fue mediante eliciting: el autor indicaba algo al informador mediante gestos y anotaba el equivalente (Cardona, 2006: 32-33). El tipo de léxico está justificado por exigencias comerciales (nombres de productos, cuantificadores...) y elementos abstractos útiles para conversar con los indígenas en cada uno de los lugares por los que fueron parando y explorando.

Podemos decir que la primera vuelta en torno al mundo fue el primer gran ejercicio de globalización de la humanidad en el que se comprobó la existencia de un estrecho que unía el Atlántico con el Pacífico y se dieron a conocer en Europa nuevas culturas ignotas hasta el momento conectando así todos los territorios de la Tierra. El relato nos reporta una gran riqueza de informaciones sobre lo que ocurrió durante los casi tres años que duró el viaje: los puertos en los que recalaron, las gentes que encontraron, las costumbres de los indígenas, las riquezas de las tierras que iban conociendo, las lenguas vernáculas, la descripción del cultivo y recolección del clavo, el jengibre, la canela y la nuez moscada.

Se trataba de una expedición con fines comerciales que aportó beneficios a muchos campos entre los cuales encontramos el de la gastronomía. Se necesitaban especias porque no existían modos de conservar los alimentos y necesitaban enmascarar los sabores, hacer más sabrosas las comidas...

La expedición quería abrir una nueva ruta comercial por Occidente hacia las Molucas, pues la ruta de Oriente estaba sobrecargada, se había vuelto peligrosa y costosa, para conseguir las preciadas especias (clavo, canela, nuez moscada y jengibre) que en aquella época, en Europa, tenían un valor comercial enorme, pues permitían la conservación de los alimentos y el encubrimiento de los sabores dado que no habiendo sistemas de refrigeración, los alimentos se echaban a perder rápidamente. Las especias, además de ser útiles como medio de conservación de alimentos, eran una fuente farmacopea por sus distintas propiedades: antisépticas, estimulantes de la digestión, antitrombóticas o antiinflamatorias.

\section{La comida en la Relación}

\section{1. ¿Aquí cuándo se come?}

En esta sección veremos en qué ocasiones se hace referencia a los alimentos y a las materias primas que sirven para la elaboración de las comidas.

\subsubsection{Avituallamiento y autoabastecimiento en las naves}

La primera referencia a la comida que hallamos en el texto, se refiere al avituallamiento de las naves cuando emprenden el viaje: ${ }^{2}$

2 Por Real Cédula de 5 de mayo de 1519, expedida en Barcelona, el Rey Carlos I dispuso el número de personas y 
[28] Salimos de San Lúcar el martes 20 de septiembre del mismo año en dirección al garbino y el 26 del mismo mes llegamos a una isla de Gran Canaria llamada Tenerife, a 28 grados de latitud, para procurarnos carne, agua y madera. [29] Estuvimos allí tres días y medio para avituallar a la flota de estas cosas [...].

La descripción y enumeración de lo que embarcan será una constante en toda la narración, cada vez que pueden realizan un avituallamiento de víveres para sobrevivir hasta el siguiente alto en el camino en tierra firme. Pero la tripulación también trató de combatir el hambre intentando procurarse sustento durante la travesía pescando todo aquello que se dejaba pescar. Y así, tratan de comer tiburones (36-37), pingüinos, para los que ni nombre tienen los europeos (99-102). ${ }^{3} \mathrm{Y}$ quizás uno de los pasajes más tristes, cuando Pigafetta cuenta que se comían el cuero de las entenas, serrín de madera, agua putrefacta y las ratas del mismo barco (239-242).

[36] Se acercaban al bordo de la nave unos peces grandes llamados tiburones que tienen dientes terribles y si encuentran hombres en el mar los devoran. [37] Pescamos muchos con anzuelos de hierro a pesar de que solo los pequeños son buenos para comer y ni siquiera son muy buenos.

[99] Proseguimos, costeando por la misma ruta, hacia el polo antártico hasta encontrar dos islas llenas de patos y lobos marinos. [100] Es de verdad imposible estimar el gran número de patos: en una hora cargamos las cinco naves. [101] Estos patos son negros y las plumas son iguales en el cuerpo y en las alas; no vuelan y se alimentan de peces. [102] Estaban tan gordos que no había que desplumarlos sino desollarlos.

[239] Estuvimos tres meses y veinte días sin probar alimento alguno. [240] Comíamos galleta que ya no era tal, sino polvo, con gusanos a puñados porque se lo habían comido prácticamente todo, y que apestaba a orines de rata, bebíamos agua amarilla putrefacta desde hacía tiempo y nos comimos el cuero que recubría la entena mayor que servía para que esta no rompiese el cabo que la sujetaba. Como estaba durísimo por el sol, la lluvia y el viento [241] lo dejábamos a remojo durante cuatro o cinco días en el mar y después lo poníamos un rato sobre las brasas para poder comerlo; muchas veces comimos el serrín de las tablas. [242] Las ratas se vendían a medio ducado cada una iy eso de haberlas tenido!

En este último párrafo se habla de los estragos que el hambre, la sed y una enfermedad nueva, el escorbuto, causada por la falta de vitamina C hicieron entre la tripulación durante la navegación

cantidad de algunas provisiones que había de llevar Magallanes en la Armada. Martín Fernández de Navarrete incluyó en el tomo IV, de su «Colección de viajes y descubrimientos que hicieron por mar los españoles desde fines del siglo XV», la relación de víveres que llevó la Armada del famoso navegante para el viaje a las islas de la especiería.

https:/ / lacritica.eu/noticia/1658/500-aniversario-de-magallanes-y-elcano/provisiones-y-alimentacion-en-lastravesias-oceanicas-del-siglo-xvi.html

3 El hecho de que Pigafetta careciera de un nombre para denominar a los pingüinos y aluda a ellos con la palabra «occati», en véneto «patos» (por analogía con estos animales) lo hemos reflejado en nuestra traducción con el equivalente literal «patos». Si hubiéramos traducido con «pingüinos» (que de todas formas especificamos en nota que se trata de estos animales) se habría perdido una importante información como es el hecho de que Pigafetta ve por primera vez estos animales. 
$\mathrm{M}^{\mathrm{a}}$ Soledad Aguilar Domingo \& $\mathrm{M}^{\mathrm{a}}$ Enriqueta Pérez Vázquez. A la mesa con Magallanes, Elcano

y Pigafetta

por el Océano Pacífico.

\subsubsection{Como acto social}

En los lugares que van visitando, la forma más común de agasajo hacia los navegantes es ofrecerles de comer (en cambio los europeos disparaban los cañones, desfilaban con sus armaduras y en más de una ocasión Pigafetta cuenta que los indígenas se asustaban muchísimo).

[392] El capitán general le invitó a cenar con nosotros y le regaló muchas cosas.

\subsubsection{Como intercambio de mercancías}

Este acto está relacionado con el avituallamiento de las naves, pues la tripulación necesitaba cargar las bodegas para seguir la navegación, pero también como forma de comercio: los navegantes intercambiaban maravillados lo que consideraban baratijas (bonetes rojos, clavos, anzuelos...) con algo fundamental para su subsistencia, la comida.

[47] Por un anzuelo para pescar o un cuchillo nos daban 5 o 6 gallinas, por un peine un par de gansos, por un espejo o unas tijeras, pescado suficiente para 10 hombres, por un cascabel o un cordel, una cesta de batatas (estas batatas saben como las castañas y son alargadas como los nabos) y por un rey de oros (que es una carta de juego) me dieron 6 gallinas creyéndose que me habían engañado.

[503] Hechas las paces, el capitán hizo dar una colación; después el príncipe y el rey regalaron al capitán, de parte de su rey, muchos cestos de arroz, cerdos, cabras y gallinas y le dijeron que les perdonase porque todas esas cosas eran pocas para uno como él. [504] El capitán regaló al príncipe un paño blanco de tela finísima, un bonete rojo, unas cuantas hebras de cuentas de cristal y un vaso dorado de vidrio.

Merece la pena comentar en el párrafo [47] donde se halla uno de los numerosos culturemas del libro y un ejemplo de la situación de interculturalidad en la que vivió su autor durante casi tres años, el paréntesis que hace Pigafetta, explicando al lector qué es un «rey de oros», algo que en la cultura española no sería necesario, pero sí para la italiana, a la que iba dirigida la Relación.

\subsubsection{Descripción de las comidas exóticas ${ }^{4}$}

La tripulación fue encontrando productos alimentarios desconocidos en Europa que despiertan la curiosidad de Pigafetta por lo que emplea buena parte de la narración en la descripción del cultivo, la extracción o el modo de elaborarla o de procurársela, la consistencia, el sabor, el olor y siempre

4Como exotismo relacionado con el comer, puede citarse aquí el ritual de la matanza del cerdo [614-628].

SCRIPTA, Revista internacional de literatura i cultura medieval i moderna, núm. 18 / desembre 2021 / pp. $182-194$ ISSN: 2340-4841 · doi:10.7203/SCRIPTA.18.22773 
$\mathrm{M}^{a}$ Soledad Aguilar Domingo \& $\mathrm{M}^{\mathrm{a}}$ Enriqueta Pérez Vázquez. A la mesa con Magallanes, Elcano

y Pigafetta

compara estos exóticos alimentos con los conocidos en Europa para tratar de describir su aspecto y su sabor. Desde el punto de vista traductivo es aquí donde los traductores han hallado mayores dificultades y también donde se observan mayores discrepancias entre las traducciones. ${ }^{5}$

[58] Comen la carne humana de sus enemigos y no porque sea buena, sino por costumbre. [59] Esta costumbre recíproca tiene su origen en una mujer anciana cuyo único hijo fue matado por sus enemigos. [60] Por este motivo, al cabo de algunos días los suyos capturaron a uno del grupo que había matado a su hijo y lo llevaron ante la vieja. [61] Ella, al verlo y recordando a su hijo, corrió hacia él y le mordió en un hombro. [62] Poco tiempo después este huyó y regresó con los suyos contando que habían intentado comérselo y les mostró la mordedura en el hombro. [63] A partir de entonces cuando capturaban prisioneros se los comían y lo mismo hacían los enemigos y de este modo se sentó la costumbre. [64] No los comen de una vez, sino que cada uno corta un trozo y lo lleva a su casa para ahumarlo. [65] Luego, cada 8 días, corta un trocito y lo come asado junto a otras cosas para recordar a sus enemigos.

[72] Hacen hogazas de pan, no muy bueno, con la médula de los árboles que se encuentra entre la madera y la corteza y se parece al requesón.

\subsubsection{Para situar la narración}

A menudo Pigafetta temporiza la narración basándose en un antes o un después de un desayuno, una comida, una merienda, una colación o una cena (antes de comer, después de cenar, antes del desayuno...). De hecho, son menos las ocasiones en las que Pigafetta usa las horas del día para situar la narración.

[485] Después de comer, vino a las naves el sobrino del rey, que era príncipe, con el rey de Mazaua [...].

[739] Después de cenar el rey hizo que trajeran una estera de cañas [...].

\subsubsection{Listas de alimentos y animales}

Si en la narración de Pigafetta existe un esquema recursivo este es una especie de estribillo que recita cuando dejan un lugar en donde se paraban, una enumeración de lo que se hallaba en aquel lugar. Esta lista de animales y alimentos rompe a veces el clímax de la narración por lo que lo más probable es que se trate de una pura transcripción de los apuntes tomados durante el viaje.

\footnotetext{
5 Véase a este respecto Aguilar Domingo; Pérez Vázquez (2020), donde se tratan algunas de las palabras que revelan mayores discrepancias entre las traducciones existentes.

SCRIPTA, Revista internacional de literatura i cultura medieval i moderna, núm. 18 / desembre 2021 / pp. 182-194 ISSN: 2340-4841 · doi:10.7203/SCRIPTA.18.22773
} 
$\mathrm{M}^{a}$ Soledad Aguilar Domingo \& $\mathrm{M}^{\mathrm{a}}$ Enriqueta Pérez Vázquez. A la mesa con Magallanes, Elcano

y Pigafetta

[715] En esta isla se encuentran perros, gatos, arroz, mijo, panizo, sorgo, jengibre, higos, naranjas, limones, caña dulce, ajo, miel, cocos, nanjeas, calabazas, carne de muchos tipos, vino de palma y oro.

[872] Allí hay canela, jengibre, mirabolanos, naranjas, limones, nanjeas, melones, pepinos, calabazas, rábanos, cebollas, escalonias, vacas, búfalos, cerdos, cabras, gallinas, ocas, ciervos, elefantes, caballos y otras cosas.

[1192] En todas las islas Malucas se encuentra clavo, jengibre, sagu (su pan hecho con madera), arroz, cabras, ocas, gallinas, cocos, higos, almendras más gordas que las nuestras, granadas dulces y agrias, naranjas, limones, batatas, miel de abejas pequeñas como hormigas (que hacen la miel en los árboles), cañas dulces, aceite de coco y de ajonjolí, melones, pepinos, calabazas, una fruta refrescante grande como la sandía llamada comulicai y otra fruta parecida al melocotón llamado guaue y otras cosas de comer.

\subsubsection{En los glosarios}

Por último, se nombra la comida en los vocabularios o glosarios que Pigafetta incluye en su libro, donde se ofrecen las equivalencias léxicas en las variedades lingüísticas que van conociendo y en italiano. El primer vocabulario es de la tierra del Verzín (lo que se correspondería con una parte del actual Brasil, el segundo, de los gigantes patagones, el tercero corresponde al léxico de los pueblos gentiles de las actuales Marianas y el último al vocabulario de los pueblos moros, en la actual Indonesia. El ejemplo que aquí ofrecemos (en 720), es del glosario de los pueblos gentiles.

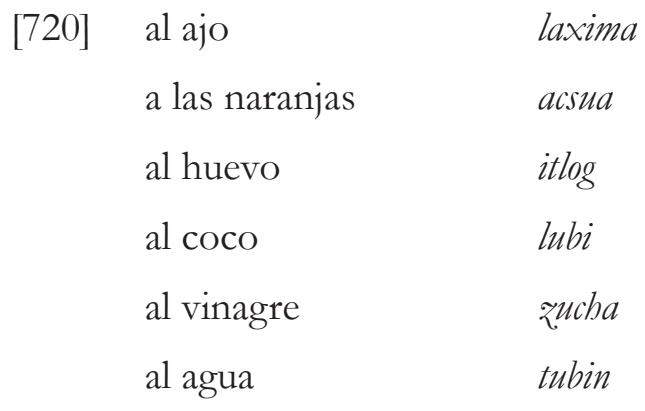

El mismo Pigafetta alude a sus apuntes para la composición de los glosarios cuando están en una de las islas del Archipiélago de San Lázaro, como se lee en [377]

[376] Antes de la hora de cenar, regalé al rey muchas cosas que le había llevado. [377] Escribí cómo 
llamaban a muchas cosas. [378] Cuando el rey y sus hombres me vieron escribir y luego leer sus palabras, se quedaron atónitos.

\section{La traducción}

En esta sección exploraremos algunos términos que han presentado problemas de interpretación o identificación y como consecuencia de traducción. De este modo, primero trataremos el equivalente en italiano moderno y luego la mejor traducción posible al español. Como ya advertimos en la introducción, los números que preceden a los párrafos son de la edición del texto que hemos traducido (Canova, 1999).

Como hemos venido viendo hasta aquí, en la Relación, Pigafetta nos habla a menudo de alimentos y comidas. Si hiciésemos una división temática de las ocurrencias podría ser la siguiente: (1) embarcadas en las naves en Sevilla (carne, galleta, vino, etc.); (2) de «emergencia» para placar el hambre, por supervivencia: cuero de las entenas, ratas, tiburones pequeños; (3) exóticas de los pueblos y lugares que iban encontrando (higos largos, cocos, sagú, vino de arroz, etc.).

Las palabras pertenecientes a los dos primeros grupos no han presentado especiales dificultades de traducción, salvo el término biscoto, al principio de la obra. Esta palabra en español se puede traducir como «bizcocho» o «galleta», dependiendo del contexto.

En nuestra traducción resultaban correctas las dos, pero nos decantamos por la palabra galleta, y no por bizcocho pues nos parecía la más adecuada dado que, aunque el lector no conociera la acepción de "pan sin levadura para los barcos» (la segunda del Diccionario de la Lengua Española, $D L E)$, «galleta» da a quien lee la percepción de algo crujiente y menos perecedero de un bizcocho.

Bizcocho De bis- y el lat. coctus 'cocido'.

1. m. Dulce blando y esponjoso, hecho generalmente con harina, huevos y azúcar, que se cuece en el horno.

2. m. Pan sin levadura, que se cocía por segunda vez para que perdiese la humedad y durase mucho tiempo.

En cambio, muchas palabras del último grupo, el que hemos denominado exotismos, nos han planteado dificultades e incluso problemas de traducción por varias razones.

\subsection{Dificultades de traducción ${ }^{6}$}

Pigafetta, viendo por primera vez determinados alimentos, no tiene un nombre para ellos y por

6 Para la clasificación de las dificultades y problemas de traducción tomamos como referencia la definición de C. Nord: «Un problema es una dificultad lingüística o extralingüística objetiva y es independiente de la capacidad del traductor, mientras que una dificultad tiene un carácter subjetivo según los conocimientos del traductor» (Nord, 1991:151). 
$\mathrm{M}^{\mathrm{a}}$ Soledad Aguilar Domingo \& $\mathrm{M}^{\mathrm{a}}$ Enriqueta Pérez Vázquez. A la mesa con Magallanes, Elcano

y Pigafetta

esta razón o los cita describiéndolos por analogía con algunos conocidos o los cita con el nombre exótico seguido de una breve descripción, como se puede ver en los siguientes ejemplos:

[282] mangiano cochi, batate, ucceli, fighi longhi uno palmo, canne dolci e pesci volatori con altre cose.

[282] Comen cocos, batatas, pájaros, higos del tamaño de una mano, caña dulce, peces voladores y otras cosas.

[300] Quando vistenno la cortesia del capitanio li presentorono pesci, uno vaso di vino de palma (che lo chiamano uraca), fighi longhi d'uno palmo e altri più picoli, più saporiti, e due cochi.

[300] Cuando vieron la amabilidad del capitán, le regalaron pescado, un recipiente con vino de palma (que llaman uraca), higos alargados, grandes como una mano y otros más pequeños, más sabrosos y dos cocos.

Los higos aparecen en la Relación 18 veces y a menudo van acompañados por los adjetivos largos o alargados. Es evidente que no se trata de higos, puesto que las higueras mediterráneas (Ficus carica) no existían por aquellas latitudes. Su identificación es una de las más estudiadas y controvertidas en la historia de la traducción de la Relación. Se piensa que Pigafetta se refiere a los diferentes tipos de bananas y plátanos (Musa acuminata, Musa balbisiana, Musa x paradisiaca) (Vargas, 2020: 168), lo que no resultaría sorprendente, dado que el antiguo nombre de los plátanos en muchos idiomas incluía esta palabra: figues de paraiso (francés), fico d'Adamo (italiano), figos (portugués), bigos de Adán (español). Por otra parte, sabemos que los plátanos o bananas fueron una comida básica para los indígenas y para los navegantes.

En el siguiente ejemplo nos habla de dos frutas, comulicai y guaue, que no han sido identificadas con un realia, pero al ir acompañadas de una breve descripción no dificultan la comprensión por parte del lector ni en italiano ni en su traducción al español. Como puede notarse en nuestra traducción, hemos decidido ser fieles a Pigafetta en su estrategia de usar la palabra indígena y glosarla con su descripción.

[1192] In tucte queste isolle de Maluco se trovano garofoli, gengero, sagu (ch'è'l suo panne di legno), riso, capre, oche, galine, cochi, fighi, mandolle più grosse de le nostre, pomi granati dolci e garbi, naranci, limoni, batate, mele de ape picolle como formiche (le qualli fanno la melle ne li arbori), canne dolci, olio de coco e de giongioli, meloni, cocomari, zuche, uno fructo refrescativo grande come le angurie deto comulicai e uno altro fructo casi como lo persico deto guaue e altre cose da mangiare.

[1192] En todas las islas Malucas se encuentra clavo, jengibre, sagu (su pan hecho con madera), arroz, cabras, ocas, gallinas, cocos, higos, almendras más gordas que las nuestras, granadas dulces y agrias, naranjas, limones, batatas, miel de abejas pequeñas como hormigas (que hacen la miel en los árboles), cañas dulces, aceite de coco y de ajonjolí, melones, pepinos, calabazas, una fruta refrescante grande como la sandía llamada comulicai y otra fruta parecida al melocotón llamado guaue y otras cosas de comer. 
$\mathrm{M}^{a}$ Soledad Aguilar Domingo \& $\mathrm{M}^{\mathrm{a}}$ Enriqueta Pérez Vázquez. A la mesa con Magallanes, Elcano

y Pigafetta

Otro ejemplo de dificultad traductiva es el término ambulon del que no ha sido identificado el realia que denomina, pero dada la descripción interna al texto se entiende perfectamente:

[1237] Vanno tosi e tuti nudi; corenno molto; hanno la voce sotille; abitano in cave soto terra e mangiano pesce e una cosa che nasce fra l'arbore e la scorsa, che è bianca e rotonda como coriandoli de confeto, deta ambulon; ma per li gran corenti de acqua e molti basi non li andasemo.

[1237] Van rasurados y completamente desnudos; corren mucho; tienen la voz fina; viven en cuevas bajo tierra y comen pescado y una cosa que nace entre el árbol y su corteza, que es blanca y redonda como una semilla de cilantro, que llaman ambulon; pero por las grandes corrientes de agua y por los bajíos, no fuimos allí.

Cuando están en el estrecho de la Patagonia, Pigafetta habla de la brevedad de las noches en octubre (tres horas), de la belleza de la zona, de los numerosos puertos ricos de agua y de la pesca abundante que allí hay y nombra tres tipos de peces: los doradi, albacore e bonniti, que no son otra cosa que «los dorados», «las albacoras» (atunes blancos) y «los bonitos» que abundan en las aguas tropicales y subtropicales. Los colondrini son las golondrinas de mar o chicharras que pertenecen al género Dactylopterus, denominadas en italiano rondini. En este párrafo Pigafetta da muestras de gran creatividad lingüística al calcar términos españoles adaptándolos a la morfología del italiano e incluso a la fonotáctica (nótese la doble -nn- de bonniti). Dado que se trata de hispanismos pigafettianos nuestra estrategia traductiva aquí ha sido la de conservar los términos, de este modo, se recreaba la babel lingüística en la que vivieron durante casi tres años.

[228] In questo Mar Occeanno se vede una molto delectevol caza de pesci: sonno tre sorte de pessi longhi uno brazo e piú che se chiamano doradi, albacore e bonniti, li qualli seguitano pesci che volanno, chiamatti colondrini, longhi uno palmo e piú e sonno obtimi al mangiare. [229] Quando quelle tre sorte trovano alguni de questi volanti, subito li volanti saltanno fora de l'acqua e volano finché hanno le alle bagnate piú de uno trar de balestra. [230] Intanto che questi volano, li altri corenno indietro socta acqua a la sua ombra; non sonno cussí presto cascati ne l'acqua che questi subito li piglianno e mangiano, cosa invero belissima de vedere.

[228] En este Mar Océano hay pesca abundante: hay tres tipos de peces de más de un brazo de longitud que se llaman doradi, albacore y bonniti. Estos peces siguen a unos peces que vuelan que se llaman colondrini y son más largos de un palmo y muy buenos para comer. [229] Cuando las tres especies anteriores encuentran peces de los que vuelan, estos últimos saltan fuera del agua y vuelan mientras tienen las alas mojadas, más allá de la trayectoria de una flecha de ballesta. [230] Mientras estos vuelan los otros van detrás de ellos siguiendo su sombra por debajo del agua. En cuanto los peces voladores caen al agua los otros los atrapan y se los comen y verlo es maravilloso.

\subsection{Problemas de traducción}

El término chiacare ha causado uno de los mayores problemas de traducción, pues no se ha identificado con absoluta certeza el realia que nombra. Tras haber consultado la bibliografía existente 
$\mathrm{M}^{\mathrm{a}}$ Soledad Aguilar Domingo \& $\mathrm{M}^{\mathrm{a}}$ Enriqueta Pérez Vázquez. A la mesa con Magallanes, Elcano

y Pigafetta

y con la ayuda de dos botánicos históricos, Pablo Vargas del CSIC y Teresa Nobre de Carvahlo, investigadora del CHAM (Center for the Humanities de Lisboa) hemos llegado a traducirlo como «nanjeas» gracias a la descripción que hace en el siguiente fragmento:

[1207] Da Sulach circa 10 leghe ala medesima via trovassemo una isola assai grande, nela qualle se trova riso, porci, capre, galine, cochi, canne dolci, sagu, uno suo mangiare de fighi [***] el qualle chiamano chanali, chiacare (a queste chiamano nangha: le chiacare sonno fructi como le angurie: de fora nodose, de dentro hanno certi fructi rossi, picoli come armelini; non hanno osso, ma per quello hanno una medola como un fazolo, ma più grande e al mangiar tenere como castagne) e un fructo facto como la pigna, de fuora iallo e bianco de dentro, e al tagliare como un pero, ma più tenero e molto megliore, deto connilicai.

[1207] Por la misma ruta, a unas 10 leguas de Sulach encontramos una isla muy grande, en la que se encuentra arroz, cerdos, cabras, gallinas, cocos, caña dulce, sagú, un alimento suyo a base de higos $\dagger \ldots \dagger$ al que llaman chanali, nanjeas (a estas les llaman nangha: las nanjeas son frutos como las sandías: por fuera nudosas, dentro tienen unos frutos rojos, pequeños como albaricoques; no tienen hueso, en su lugar tienen un corazón como una judía, pero un poco más grande y tierno como la castaña) y un fruto parecido a la piña, por fuera amarillo y blanco por dentro, cuando se corta parece una pera, pero más tierno y mucho más bueno, llamado comulicai.

Según la botánica histórica Teresa Nobre, posiblemente se esté refiriendo a «jaca», el nombre portugués de un fruto asiático cuyo nombre local es Chakka (Arthocarpus integrifolia L. I Artocarpus heterophyllus), de la familia de las moráceas, al que ya se habían referido otros viajeros europeos, cronistas y médicos del siglo XVI como Niccolò de' Conti y Ludovico de Vartema.

Otro de los términos que nos ha causado más quebraderos de cabeza es missiglioni. La expedición se encuentra en el Puerto de San Julián y Pigafetta nos habla de unos moluscos a los que llama missiglioni (palabra inventada por el autor derivada de la adaptación fonética de la voz española «mejillones», pues la traducción al italiano actual es cozze). Sin embargo, es muy improbable que se tratase de mejillones, puesto que en la descripción del molusco, Pigafetta añade que tienen perlas en su interior, como se ve en [186].

[186] In questo porto era asaissime cape longhe, che le chiamano missiglioni (avevano perle nel mezo), ma picole che non le potevano mangiare.

[186] En este puerto hay una gran cantidad de conchas pequeñas y alargadas a las que llaman missiglioni, que llevan perlas dentro, pero tan pequeñas que no las podíamos comer.

Podríamos pensar que Pigafetta no conociera las ostras, y se refiriese a ese animal con el nombre de missiglione por su relativo parecido, pero más adelante nos deja claro que no es así, pues hace referencia a ostrighe (ostras): 
$\mathrm{M}^{a}$ Soledad Aguilar Domingo \& $\mathrm{M}^{\mathrm{a}}$ Enriqueta Pérez Vázquez. A la mesa con Magallanes, Elcano

y Pigafetta

[885] Gli sonno cocodrili grandi cussì de terra como de mare, ostrighe e cape de diverse sorte, fra le altre no trovassemo due: la carne del'una pezò vintisei libre e l'altra quarantacatro.

[885] Hay cocodrilos grandes tanto de tierra como de mar, ostras y moluscos de varios tipos. Entre otros encontramos dos, la carne de uno pesaba veintiséis libras y la del otro cuarenta y cuatro.

\section{Conclusiones}

En este breve trabajo hemos presentado las ocasiones en las que Antonio Pigafetta habla, alude, describe o diserta sobre la comida o las materias primas para producirla. Tanto nos parece que está presente este campo semántico y léxico, que estaríamos tentadas de hablar de la primera circunnavegación como una expedición de carácter gastronómico. ${ }^{7}$ Sin embargo, la expedición tuvo sobre todo una finalidad comercial, territorial y política como demuestra la gran cantidad de bibliografía publicada sobre el tema. La Relación refleja lo fundamental que resulta para los seres vivos la comida para sobrevivir, como fuente de energía y buena salud, como forma de socialización, de intercambio y como muestra de identidad cultural. Hemos mostrado con algunos ejemplos las dificultades y estrategias de traducción utilizadas en la próxima edición bilingüe de la Relazione del primo viaggio attorno al mondo - Relación del primer viaje en torno al mundo, que coincidirá con el quinto centenario de la llegada de la nave Victoria tras la primera circunnavegación del mundo.

7 Tal tentación viene alimentada por la siguiente afirmación de Vargas (2020: 177): «La mayoría de las citas corresponden a plantas (58\%), lo que indica que se trataba de una expedición de carácter botánico». 
$\mathrm{M}^{\mathrm{a}}$ Soledad Aguilar Domingo \& $\mathrm{M}^{\mathrm{a}}$ Enriqueta Pérez Vázquez. A la mesa con Magallanes, Elcano

y Pigafetta

\section{Bibliografía}

Aguilar Domingo, M. S.; Pérez Vázquez, M. E. (2020) «Problemas de interpretación léxica en la traducción del manuscrito de Antonio Pigafetta», Artifara: Revista de lenguas y literaturas ibéricas y latinoamericanas.

Aguilar Domingo, M.S.; Canova, A.; Pérez Vázquez, M.E.; Valladares Ramírez, R. (en prensa) Relazione del primo viaggio attorno al mondo. Relación del primer viaje en torno al mundo, Madrid, Polifemo.

Canova, A. (ed.) (1999) Relazione del primo viaggio attorno al mondo, Padova, Editrice Antenore.

Cardona, G. R. (2006) Introdurione all'Etnolinguistica, Torino, UTET.

Corominas, J. (1973 [2016]) Breve Diccionario etimologico de la lengua castellana, Madrid, Gredos, 2016.

DEI: Dizionario Etimologico Italiano (1950-1957) Carlo Battisti; Giovanni Alessio, Firenze, Barbera Editore.

DGLC: Diccionario general de la lengua castellana (1849) José Caballero; Cipriano de Arnedo, Madrid, Imprenta de la Viuda de D. R. J. Domínguez.

DLE: Diccionario de la lengua española, Real Academia Española, 23. ${ }^{a}$ ed., [versión 23.3 en línea]. $<$ https://dle.rae.es $>$ [agosto-octubre 2020].

DN: Diccionario Nacional ó Gran Diccionario Clásico de la Lengua Española (1846) Ramón Joaquín Domínguez, Madrid: Establecimiento léxico-tipográfico de R. J. Domínguez.

Masoero, M. (ed.) (1987) Viaggio attorno al mondo di Antonio Pigafetta, Rovereto, Longo Editore.

Nord, C. (1991) Text Analysis in Translation. Theory, Methodology and Didactic Application of a Model for Translation-Oriented Text Analysis, Amsterdam, Rodopi.

O’Scalan, Timoteo et al. (1931) Diccionario Marítimo Español, Madrid, Imprenta.

Treccani Enclicopedia www.treccani.it/enciclopedia

Vargas Gómez, P. (2020) «Análisis de la historia natural del diario de Pigafetta», En búsqueda de las especias. Las plantas de la expedición Magallanes-Elcano (1519-1522), P. Vargas Gómez (ed.), Madrid, CSIC, pp. 161-180. 